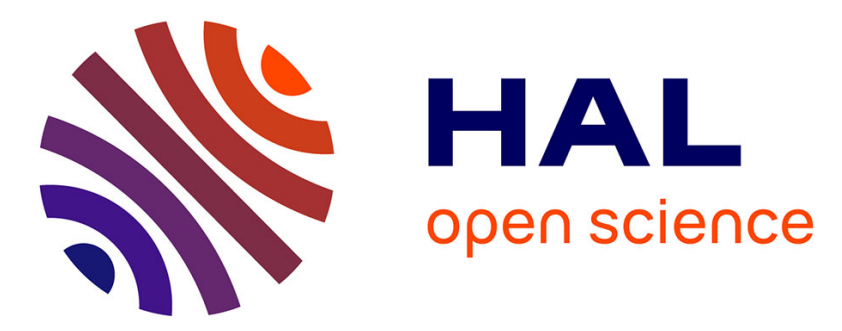

\title{
Stability analysis of within-host parasite models with delays.
}

Abderrahman Iggidr, Joseph Mbang, Gauthier Sallet

\section{To cite this version:}

Abderrahman Iggidr, Joseph Mbang, Gauthier Sallet. Stability analysis of within-host parasite models with delays.. Mathematical Biosciences, 2007, 209 (1), pp.51-75. 10.1016/j.mbs.2007.01.008 . inria00552051

\section{HAL Id: inria-00552051 \\ https://hal.inria.fr/inria-00552051}

Submitted on 5 Jan 2011

HAL is a multi-disciplinary open access archive for the deposit and dissemination of scientific research documents, whether they are published or not. The documents may come from teaching and research institutions in France or abroad, or from public or private research centers.
L'archive ouverte pluridisciplinaire HAL, est destinée au dépôt et à la diffusion de documents scientifiques de niveau recherche, publiés ou non, émanant des établissements d'enseignement et de recherche français ou étrangers, des laboratoires publics ou privés. 


\title{
Stability analysis of within-host parasite models with delays
}

\author{
Abderrahman Iggidr ${ }^{\mathrm{a}, *}$ Joseph Mbang ${ }^{\mathrm{a}, \mathrm{b}}$ Gauthier Sallet ${ }^{\mathrm{a}}$ \\ a INRIA-Lorraine and University Paul Verlaine-Metz LMAM-CNRS UMR 7122, \\ ISGMP Bat. A, Ile du Saulcy 57045 Metz Cedex 01, F \\ ${ }^{\mathrm{b}}$ Department of mathematics, University of Yaoundé I, Cameroon.
}

\begin{abstract}
We provide a global analysis of systems of within-host parasitic infections. The systems studied have parallel classes of different length of latently infected target cells. These systems can also be thought as systems arising from within-host parasitic systems with distributed continuous delays. We compute the basic reproduction ratio $\mathcal{R}_{0}$ for the systems under consideration. If $\mathcal{R}_{0} \leq 1$ the parasite is cleared, if $\mathcal{R}_{0}>1$ and if a sufficient condition is satisfied we conclude to the global asymptotic stability (GAS) of the endemic equilibrium. For some generic class of models this condition reduces to $\mathcal{R}_{0}>1$. These results make possible to revisit some parasitic models including intracellular delays and to study their global stability.
\end{abstract}

Key words: Nonlinear dynamical systems; parasite models; global stability; delays.

\section{Introduction}

The primary objective of this paper is to provide a stability analysis of withinhost parasite models dynamics with continuously distributed delays. We consider micro-parasite (virus, bacterium, protozoan) having target cells in the host. This is the case for instance for HIV, HBV or plasmodium. The parasite needs some target cells to proliferate : Lymphocyte T cells for HIV-1, Erythrocytes for plasmodium. The standard within-host parasite models have

* Corresponding author: e-mail: iggidr@loria.fr, iggidr@math.univ-metz.fr 
similar form:

$$
\left\{\begin{array}{l}
\dot{x}=\varphi(x)-\beta x v, \\
\dot{y}=\beta x v-\mu_{y} y, \\
\dot{v}=r \mu_{y} y-\mu_{v} v-u \beta x v .
\end{array}\right.
$$

In this system $x$ is the volume density of the susceptible target cells, $y$ is the density of parasitized cells, and $v$ is the density of the free parasites. The function $\varphi(x)$ describe the population dynamics of non-parasitized target cells. Two classical formulas for $\varphi$ are $\varphi(x)=\Lambda-\mu_{x} x$ and $\varphi(x)=\Lambda+a x\left(1-\frac{x}{K}\right)$. The parameter $u$ takes only the values 0 or 1 . When $u=1$ the model assumes that the parasite disappears when infecting a target cell. In some models this effect is neglected and $u=0$.

These parasitic models have been developed by many authors. With $u=1$ and a logistic population dynamics [1,2], with $u=0$ and a logistic population dynamics [3] for HIV-1 infection, with $u=0$ and $\varphi(x)=\Lambda-\mu_{x} x$ [4,5,6,7,8,9] for HIV-1 and malaria infections. For HIV and HBV models, Perelson and Nelson [3] and Nowak and May [10] provide excellent reviews and many more references. A review of intra-host models of Malaria has been done by Dietz and Molineaux [11, see the numerous references therein.

The entry of a parasite into a target-cell initiates a cascade of events that ultimately lead to the production of new parasites by the infected cell. The previous model (1) assumes this process to occur instantaneously: as soon as a parasite enters a target cell, this one begins to produce parasites. This is not biologically sensible. Therefore models with delays have been considered [12,13]. Two kinds of delays are encountered in the literature, namely discrete constant delays and continuously distributed delays. It is advocated in [14] that fixed delays are not biologically realistic, and in the context of compartmental systems continuous probability functions of lags are far more important than discrete lags [12. Usually the probability density function (pdf) of the lag is not well known and approximations are used. A widely used family of functions is given by the family of gamma distributions. Using a continuous distributed lag with a general kernel function $g$ the following model has been proposed in [15].

$$
\left\{\begin{array}{l}
\dot{x}=\varphi(x)-\beta x v, \\
\dot{y}=\beta \int_{0}^{\infty} x(t-\tau) v(t-\tau) g(\tau) e^{-m \tau} d \tau-\mu_{y} y, \\
\dot{v}=r \mu_{y} y-\mu_{v} v-u \beta x v .
\end{array}\right.
$$

In this model (2) it is assumed that the infected cell start to produce parasites $\tau$ time units after initial infection. The value of $\tau$ varies according to a probability density function $g$. The term $e^{-m \tau}$ accounts for cells infected at time $t$ but that die before producing parasites. In [14,15] the function $g$ is chosen as a gamma distribution. More complex functions can also be used. For example 
a convex combination of gamma distributions or some other more complex combinations [16,17,12]. Another alternative is to put the integral term as a source term in the compartment of the "free parasites" to obtain

$$
\left\{\begin{array}{l}
\dot{x}=\varphi(x)-\beta x v \\
\dot{v}=r \mu_{y} \beta \int_{0}^{\infty} x(t-\tau) v(t-\tau) g(\tau) e^{-m \tau} d \tau-\mu_{v} v-u \beta x v
\end{array}\right.
$$

In this case $g(\tau) e^{-m \tau}$ is interpreted as the expected production of parasites released by a cell $\tau$ time units after initial invasion by the parasite.

When $g$ is a gamma function or a convex combination of gamma distributions the system (2) can be converted into a system of differential equations. This has been used in [14. The process of converting time-delay integrodifferential equations in a set of ODE is coined by MacDonald as the "linear chain trick" [18]. In other communities this is also known as the method of stages [19,20,21,22,23]. Any distribution can be approximated by a combination of stages in series and in parallel [17,19]. Actually it can be proved that given any distribution $g$ with support on $[0, \infty)$, there is a sequence of convex combination of gamma distributions which converges weakly to this distribution. If $g$ is continuous, weak convergence implies uniform convergence on compact intervals. Least squares approximation on a finite interval is used in [12].

In this paper we will analyze the stability of models arising from system (1) by the linear chain trick. We will consider inserting between the compartment of susceptible target cells and the compartment of free parasites a system constituted by a number $q$ of parallel chains of different lengths. An infected cell enters the first compartment of the $i^{\text {th }}$-chain with probability $\pi_{i}$, with $\sum_{i=1}^{q} \pi_{i}=1$. For $q$ parallel chains, if the $i^{t h}$-chain is a cascade series of $k_{i}$ stages, we get the following model.

$$
\left\{\begin{array}{l}
\dot{x}=\varphi(x)-\beta x v \\
\text { and for } i=1, \cdots q, \\
\dot{y}_{1, i}=\pi_{i} \beta x v-\alpha_{1, i} y_{1, i}, \\
\dot{y}_{2, i}=\gamma_{1, i} y_{1, i}-\alpha_{2, i} y_{2, i}, \\
\cdots \\
\dot{y}_{k_{i}, i}=\gamma_{k_{i-1}, i} y_{k_{i-1}, i}-\alpha_{k_{i}, i} y_{k, i}, \\
\dot{v}=\sum_{i=1}^{q} \gamma_{k_{i}, i} y_{k_{i}, i}-\mu_{v} v-u \beta x v .
\end{array}\right.
$$

Usually in the linear chain trick the coefficients in the stages in series are all equal, i.e., $\alpha_{j, i}=\gamma_{j, 1}=a$ for all $j=1 \cdots k_{i}$. Here we shall not assume that these coefficients are equal. We consider that the inflow and outflow in 
each compartment are not necessarily equal. This means that our models are not compartmental models. This is not only for the sake of generality but we will need this refinement in our applications. For instance the application of the linear chain trick to the HIV-model of [15] gives a system for which the coefficients are different.

The paper is organized as follows. In Section 2 we revisit the linear chain trick and we settle some notations. In Section 3 we apply the linear chain trick to the general within-host parasitic model. The notations used in the sequel are specified and the hypotheses made on the model are stated. We compute the equilibria and the basic reproduction ratio $\mathcal{R}_{0}$ [24 25] of these models. The existence (in the positive orthant) of an endemic equilibrium is intimately related to $\mathcal{R}_{0}$. In Section 4 we provide a stability analysis when there is only one cascade chain for the system. This section is preparing the general case. The Section 5 is devoted to the general system. We prove that the parasite free equilibrium is globally asymptotically stable (GAS) if $\mathcal{R}_{0} \leq 1$. When $R_{0}>1$ we give a sufficient condition for the GAS of the endemic equilibrium. For systems for which $u=0$ (i.e. the disappearance of the parasite when entering the target cell is neglected) and $\varphi(x)=\Lambda-\mu_{x} x$, this condition is automatically satisfied, hence $\mathcal{R}_{0}>1$ is a necessary and sufficient (NSC) for the GAS of the infected equilibrium. In Section 6 we apply the preceding results to models studied by Nelson and Perelson in [15]. We come across the results of these authors and improve some of them.

\section{The general chain trick}

For the analysis of the system (4) we will need some notations. We also provide for the convenience of the reader a short proof, in a "control system theory" spirit, of what we call the general linear chain trick. Usually the linear chain trick consist in inserting a single linear chain to replicate a gamma distribution. To obtain convex combination of gamma distributions it is necessary to consider parallel chains with different lengths. The result is not new but to our knowledge the presentation is probably original, although with the abundance of related literature it is difficult to be sure.

We consider a dynamic system where a peculiar one dimensional feedback $u(x)$ has been distinguished. For example the $x v$ term appearing in the second equation of the system (1) can be viewed as a feedback.

$$
\dot{x}=f(x, u(x))
$$

The function $f$ is an application from $\mathbb{R}^{n} \times \mathbb{R}$ into $\mathbb{R}^{n}$. The function $u$ is defined from $\mathbb{R}^{n}$ to $\mathbb{R}$. The functions $f$ and $u$ are supposed to satisfy conditions that 
ensure existence and uniqueness of solutions for system (5). Usually a system $\dot{x}=f(x, u)$ is called a controlled system.

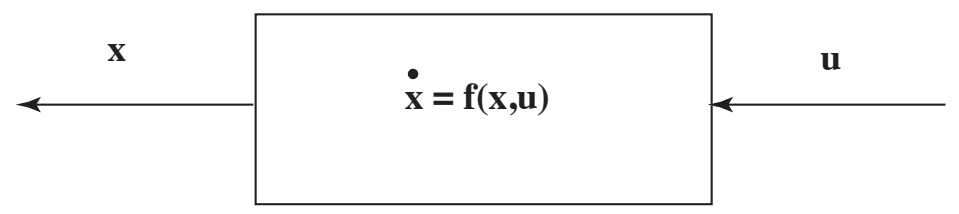

Fig. 1. Control system

When the function $u$ depends only on time $t$ it is called a control or an input. When $u$ depends on $x(t)$ it is called a feedback.

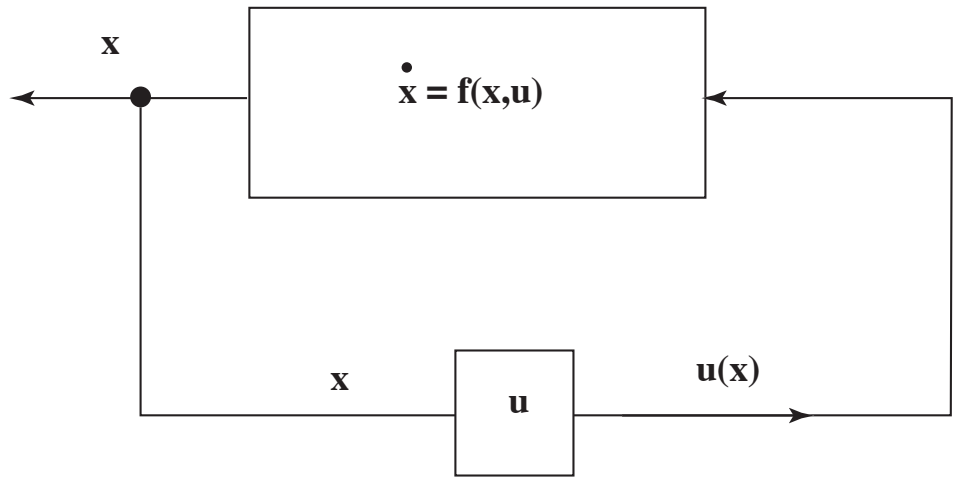

Fig. 2. Feedback

Let us consider the following controlled linear system (in control theory's sense $[26,27])$.

$$
\left\{\begin{array}{l}
\dot{y}=A y+w B, \\
z=C y
\end{array}\right.
$$

Where the state is $y \in \mathbb{R}^{k}$, the matrix $A$ is a $k \times k$ real matrix, $w$ is a real function, $B$ is a $k \times 1$ column vector, $z \in \mathbb{R}$ and $C$ is a $1 \times k$ row vector. In control theory $w$ is the input (or control), $z$ is the output (or observation). We denote by $Y(t)$ the Heaviside function, $Y(t)=0$ if $t<0$ and $Y(t)=$ 1 otherwise. This function is also known in control theory as the unit step function. For an initial state $y(0)=y_{0}$, and for a control signal $h(t)$, the output signal of (6) is given by (see for example [28])

$$
\begin{aligned}
z(t) & =C e^{t A} y_{0}+\int_{0}^{t} C e^{(t-\tau) A} B h(\tau) d \tau \\
& =C e^{t A} y_{0}+\int_{-\infty}^{t} C e^{(t-\tau) A} B h(\tau) Y(\tau) d \tau \\
& =C e^{t A} y_{0}+\int_{0}^{+\infty} C e^{\tau A} B h(t-\tau) d \tau \\
& =C e^{t A} y_{0}+C e^{t A} B Y \star h .
\end{aligned}
$$


The output is obtained by a convolution integral, where by misuse of language we have denoted by $C e^{t A} B Y(t)$ the function $t \longmapsto C e^{t A} B Y(t)$. This function is called the impulse response of the system. The reason is that this is the response of the system when the control is the Dirac function considered as a distribution ( L. Schwartz's generalized functions, [39] ). The output is obtained by convolution of the impulse response with the input. By the classical theory of ODE, $C e^{t A} B$ is then a linear combination of functions of type $t^{k} e^{\lambda t}$, $t^{k} e^{\lambda t} \cos (\omega t)$ and $t^{k} e^{\lambda t} \sin (\omega t)$ for $k \in \mathbb{N}, \lambda \in \mathbb{R}$ and $\omega \in \mathbb{R}$.

We assume that the kernel function (or the probability density function) $h$ of a certain delay can be represented by $h(t)=C e^{t A} B Y(t)$. The presence of $Y(t)$ is to ensure that the time delay is always positive. Moreover since $h(t)$ is a distribution the matrix $A$ must be a stable matrix. We can assume, without loss of generality that $B$ and $C$ are nonnegative vectors and that the off-diagonal entries of $A$ are nonnegative (which implies $e^{t A}$ is nonnegative). In other words $A$ is a Metzler stable matrix [29,27].

When there is a distributed delay (associated to the preceding $h$ ) on the feedback, the system (5) becomes an integro-differential equation:

$$
\begin{aligned}
\dot{x} & =f\left(x, \int_{0}^{\infty} u(x(t-\tau)) h(\tau) d \tau\right) \\
& =f\left(x, \int_{-\infty}^{t} u(x(\tau)) h(t-\tau) d \tau\right) .
\end{aligned}
$$

We consider an initial condition

$$
u(x(t))=\theta(t) \text { for } t \leq 0
$$

where $\theta$ is a continuous function defined on $)-\infty, 0]$.

Now we define

and

$$
y(t)=\int_{-\infty}^{t} e^{(t-\tau) A} B u(x(\tau)) d \tau
$$

$$
y_{0}=y(0)=\int_{-\infty}^{0} e^{(-\tau) A} B u(x(\tau)) d \tau=\int_{-\infty}^{0} e^{(-\tau) A} B \theta(\tau) d \tau .
$$

We have the relation

$$
\int_{-\infty}^{t} u(x(\tau)) h(t-\tau) d \tau=\int_{-\infty}^{t} C e^{(t-\tau) A} B u(x(\tau)) d \tau=z(t)=C y(t) .
$$

Therefore, for any initial state $x(0)=x_{0}$ and an initial condition $u(t)=\theta(t)$ on $\mathbb{R}_{-}$, the integro-differential equation $(8)$ is equivalent to

$$
\left\{\begin{array}{l}
\dot{x}=f(x, C y), \\
\dot{y}=A y+u(x) B,
\end{array}\right.
$$


with initial conditions $x(0)=x_{0}$ and $y(0)=y_{0}$. More precisely any solution of (8) becomes a solution of (10) (see [30] for example).

The general linear chain trick allows to replace system with distributed delays whose distributions are linear combinations of gamma functions, by a system of ODE. Realization theory is a part of linear control theory which gives explicit tools to construct such a matrix $A$ when the distribution is known. The delay is obtained by inserting between the feedback and the original system a linear system.

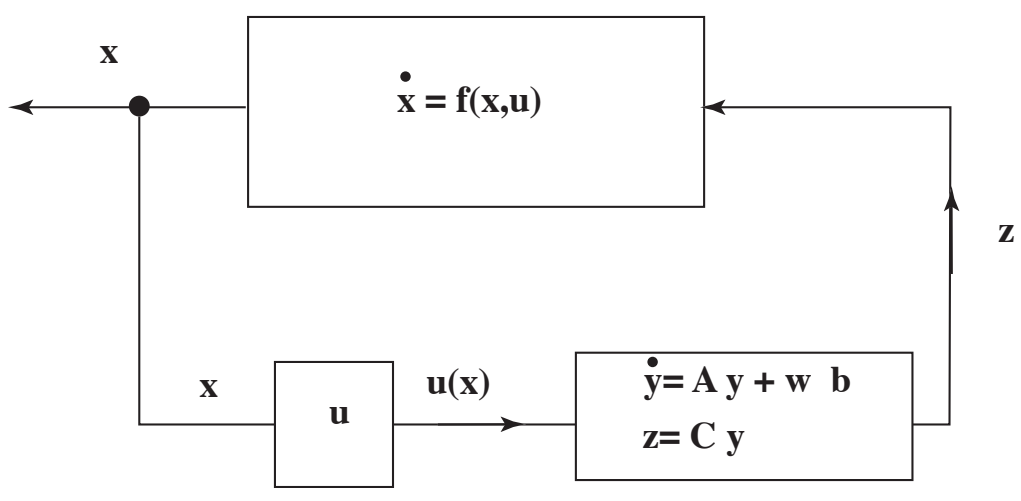

Fig. 3. Linear chain trick

Among these distributions the gamma distribution, a widely used tunable distribution, or more exactly Erlang's distribution is defined by

$$
g_{n, \sigma}(t)=\frac{t^{n-1}}{(n-1) !} \frac{1}{\sigma^{n}} e^{-\frac{t}{\sigma}}
$$

This distribution is realized by the $n \times n$ matrix $A=\frac{1}{\sigma}(N-I)$ where $I$ is the identity matrix and $N$ is the nilpotent matrix with all entries 0 excepted the sub-diagonal which is composed of 1 .

$$
N=\left[\begin{array}{cccccc}
0 & 0 & 0 & 0 & \cdots & 0 \\
1 & 0 & 0 & 0 & \cdots & 0 \\
0 & 1 & 0 & 0 & \cdots & 0 \\
\vdots & \ddots & \ddots & \ddots & \ddots & \vdots \\
0 & 0 & \cdots & 0 & 1 & 0
\end{array}\right]
$$

$B$ is the first vector of the canonical basis of $\mathbb{R}^{n}, B=[1,0, \cdots, 0]^{T}$, and $C$ is the transpose of the last vector of the canonical basis multiplied by $\frac{1}{\sigma}$, $C=\frac{1}{\sigma}[0, \cdots, 0,1]$. 


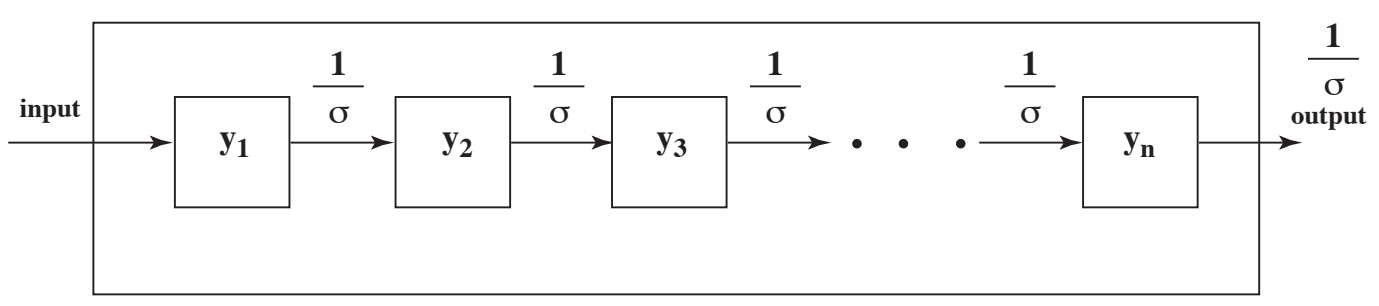

Fig. 4. realization of Erlang distribution

When the distribution $g$ is a convex combination of Erlang distributions

$$
g=\sum_{i=1}^{q} \pi_{i} g_{k_{i}, \sigma_{i}}
$$

with $\pi_{i} \geq 0$ and $\sum \pi_{i}=1$, and the corresponding delay is applied to the general class of within-host parasite models (1), the system can be replaced by the system (4). The figure (5), analogous to figure 9 of [12] represents the block diagram for the interconnection of the different systems: 


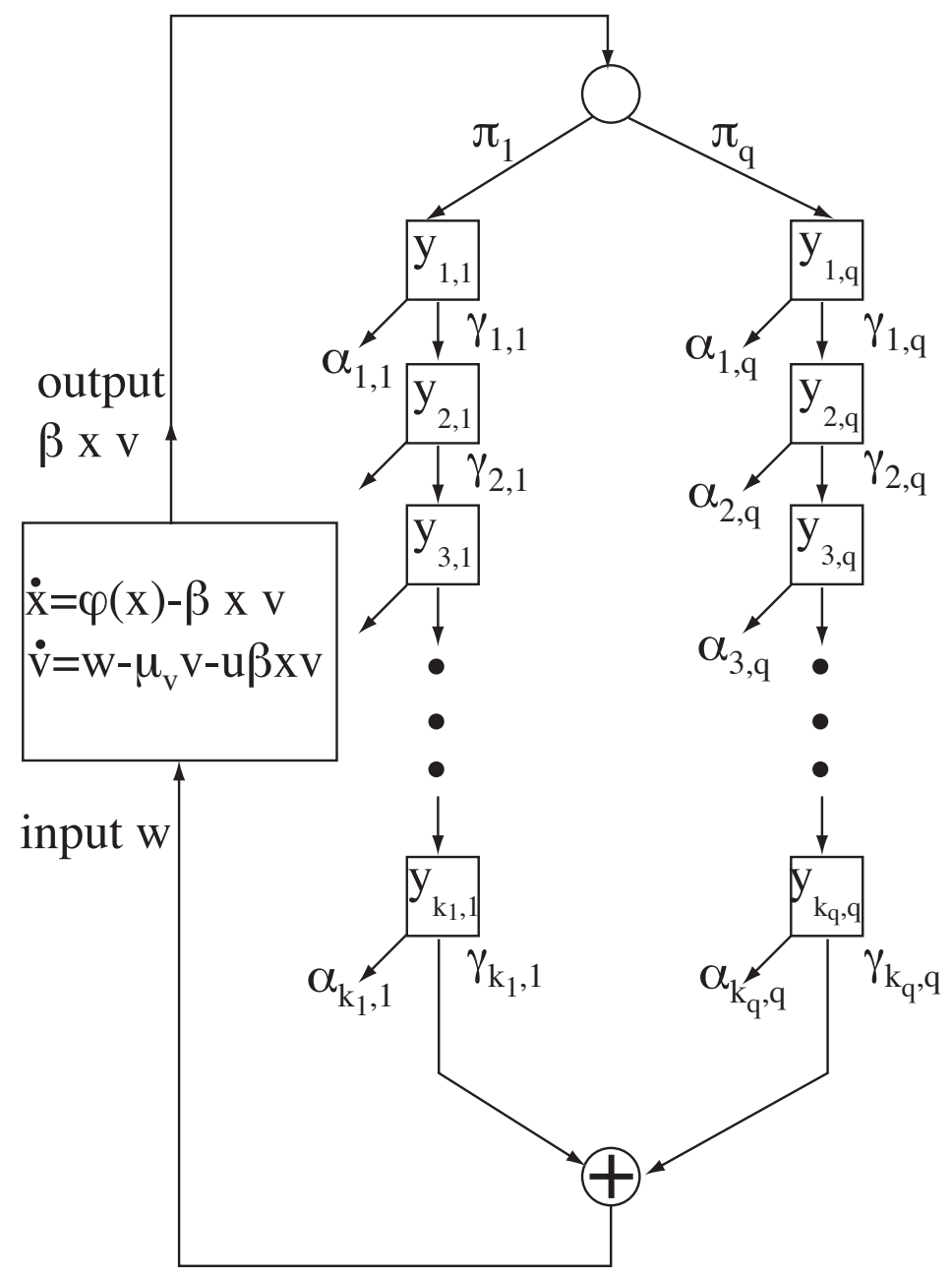

Fig. 5. Block diagram of system (4)

We draw reader's attention to the fact that this block diagram is not strictly speaking a compartmental model, since this is not a mass-balance system. From a pseudo-compartment $j$ is globally leaving a quantity $\alpha_{j} y_{j}$ of material, and entering $\gamma_{j-1} y_{j-1}$. This $\gamma_{j-1} y_{j-1}$ quantity entering in the $j$ compartment has not to be subtracted to the amount of material of the $j-1$ compartment. The coefficients $\gamma_{j}$ can be considered as yield coefficients. We only assume that the coefficients are positive. The arrows are only to symbolize what material is entering (or leaving). This picture is more a signal flow graph in control theory's spirit. To obtain an equivalence to system (2) the parameters must be settled to appropriate values as we will see later on. 


\section{Notations, background and hypotheses}

The system (4) can be written in a condensed form:

$$
\left\{\begin{array}{l}
\dot{x}=\varphi(x)-\beta x v \\
\dot{y}=A y+\beta x v B \\
\dot{v}=C y-\mu_{v} v-u \beta x v .
\end{array}\right.
$$

\subsection{Notations}

To simplify the exposition we need some notations. We will adopt some convenient notations from MATLAB or SCILAB. Matrices will be represented by entries between brackets, listed by rows, each element is separated by commas and the semicolon indicates end of the rows. We denote by $e_{k}(n)$ the $k^{t h}$-vector of the canonical basis of $\mathbb{R}^{n}$. In other words for example the vector $e_{1}(n)$ is the column vector of length $n$ written with our notations $e_{1}(n)=[1 ; 0 ; \cdots ; 0]$. We will use the notation $e_{e n d}(n)$ for the last vector of the canonical basis. We use the same convention to define block matrices, for example $M=[E, F ; G, H]$ is the block matrix

$$
M=\left[\begin{array}{ll}
E & F \\
G & H
\end{array}\right]
$$

provided that the matrices $E, F, G$ and $H$ have compatible dimensions. We denote by $A^{T}$ the transpose of the matrix $A$. For a vector $x$ of length $n$ we denote by $\operatorname{diag}(x)$ the $n \times n$ diagonal matrix with the elements of $x$ on the diagonal. We also consider $\operatorname{diag}\left(A_{1}, \cdots, A_{n}\right)$ which is a diagonal block matrix, the $A_{i}$ being the diagonal blocks.

We can now define $A, B$ and $C$ of $(12)$. The matrix $A$ is a $n \times n$ diagonal block matrix with $n=\sum_{i=1}^{q} k_{i}: A=\operatorname{diag}\left(A_{1}, \cdots, A_{q}\right)$. The $k_{i} \times k_{i}$ block $A_{i}$ is

$$
A_{i}=\left[\begin{array}{cccccc}
-\alpha_{1, i} & 0 & 0 & \cdots & 0 & 0 \\
\gamma_{1, i} & -\alpha_{2, i} & 0 & \cdots & 0 & 0 \\
0 & \gamma_{2, i} & -\alpha_{3, i} & \cdots & 0 & 0 \\
\vdots & \ddots & \ddots & \ddots & \vdots & \vdots \\
0 & \cdots & 0 & \gamma_{k_{i}-2, i} & -\alpha_{k} & 0 \\
0 & \cdots & 0 & 0 & \gamma_{k_{i}-1, i} & -\alpha_{k_{i}, i}
\end{array}\right] .
$$


The vector $B$ is the column vector of length $n$

$$
B=\left[\pi_{1} e_{1}\left(k_{1}\right) ; \pi_{2} e_{1}\left(k_{2}\right) ; \ldots ; \pi_{q} e_{1}\left(k_{q}\right)\right] .
$$

The matrix $C$ is a $1 \times n$ row vector

$$
C=\left[\gamma_{k_{1}, 1} e_{\text {end }}\left(k_{1}\right)^{T}, \gamma_{k_{2}, 2} e_{\text {end }}\left(k_{2}\right)^{T}, \ldots, \gamma_{k_{q}, q} e_{\text {end }}\left(k_{q}\right)^{T}\right] .
$$

The block decompositions of $A, B$ and $C$ are compatible.

\subsection{Hypotheses}

We start to analyze the system with minimal hypotheses on $\varphi$ but nevertheless plausible from the biological point of view. The function $\varphi(x)$ describes the population dynamics of target cells in the absence of parasites. The target cells have a finite lifetime. The function $\varphi$ models in some way homeostasis. We assume that $\varphi$ is a $\mathcal{C}^{1}$ function. Since homeostasis is maintained we assume the system

$$
\dot{x}=\varphi(x)
$$

has a globally asymptotically stable equilibrium $x^{*}>0$, that is,

$$
\varphi\left(x^{*}\right)=0, \varphi(x)>0 \text { for } 0 \leq x<x^{*}, \text { and } \varphi(x)<0 \text { for } x>x^{*} .
$$

\section{Stability analysis for the one chain system}

Before studying the general case we will examine the system given by a single chain of $k$ elements. We will use the computations of this special case to study the complete system (4). In the case of a single chain the system is reduced to

$$
\left\{\begin{array}{l}
\dot{x}=\varphi(x)-\beta x v, \\
\dot{y}=A y+\beta x v B, \\
\dot{v}=C y-\mu_{v} v-u \beta x v .
\end{array}\right.
$$

with

$$
A=\left[\begin{array}{ccccc}
-\alpha_{1} & 0 & 0 & \cdots & 0 \\
\gamma_{1} & -\alpha_{2} & 0 & \cdots & 0 \\
0 & \gamma_{2} & -\alpha_{3} & \cdots & 0 \\
\vdots & \ddots & \ddots & \ddots & \vdots \\
0 & \cdots & 0 & \gamma_{k-1} & -\alpha_{k}
\end{array}\right]
$$




$$
C=\gamma_{k} e_{\text {end }}(k)^{T} \text { and } B=e_{1}(k)
$$

It is clear that the nonnegative orthant is positively invariant by (14). The matrix $A$ is a stable Metzler matrix.

\subsection{Background}

For later references we need the expression of the nonnegative matrix $\left(-A^{-1}\right)$ ( $A$ is Metzler stable ). Let $I$ be the identity matrix. Using the fact that $A=$ $-D+N$ where $D$ is the diagonal matrix $D=\operatorname{diag}\left(\alpha_{1}, \cdots, \alpha_{k}\right)$, and $N$ is the nilpotent matrix $N=A+D$, we have $(-A)^{-1}=D^{-1}\left(I-N D^{-1}\right)^{-1}$. Since $N D^{-1}$ is nilpotent we get

$$
\left(I-N D^{-1}\right)^{-1}=I+N D^{-1}+\left(N D^{-1}\right)^{2}+\cdots+\left(N D^{-1}\right)^{k-1} .
$$

Finally,

$$
-A^{-1}=\left[\begin{array}{ccccc}
\frac{1}{\alpha_{1}} & 0 & 0 & \ldots & 0 \\
\frac{\gamma_{1}}{\alpha_{1} \alpha_{2}} & \frac{1}{\alpha_{2}} & 0 & \cdots & 0 \\
\frac{\gamma_{1} \gamma_{2}}{\alpha_{1} \alpha_{2} \alpha_{3}} & \frac{\gamma_{2}}{\alpha_{2} \alpha_{3}} & \frac{1}{\alpha_{3}} & \ldots & 0 \\
\vdots & \ddots & \ddots & \ddots & \vdots \\
\frac{\gamma_{1} \cdots \gamma_{k-1}}{\alpha_{1} \cdots \alpha_{k}} & \cdots & \ldots & \frac{\gamma_{k-1}}{\alpha_{k-1} \alpha_{k}} & \frac{1}{\alpha_{k}}
\end{array}\right] .
$$

The matrix $-A^{-1}$ is a lower triangular matrix, the $i$-term on the diagonal is given by $\frac{1}{\alpha_{i}}$, the entry $(i, j)$ with $i>j$ is

$$
\left(-A^{-1}\right)(i, j)=\frac{\gamma_{j} \cdots \gamma_{i-1}}{\alpha_{j} \cdots \alpha_{i-1}} \frac{1}{\alpha_{i}}
$$

If we use the usual convention that an empty product has value 1 , this expression is also valid for the entries on the diagonal.

\subsection{Basic reproduction ratio and Equilibria of the system}

As usual the basic reproduction number is the expected number of secondary cases produced in a completely susceptible population, by a typical infected individual during its entire period of infectiousness [31,24,32,25]. From the structure of the system the computation of $\mathcal{R}_{0}$ is straightforward. Indeed one parasite during the mean duration of its life generates a Dirac input $\frac{\beta x^{*}}{\mu_{v}+u \beta x^{*}}$ in the second controlled system $\dot{y}=A y+w B$. Hence this input generates 
secondary cases given by the formula

$$
\frac{\beta x^{*}}{\mu_{v}+u \beta x^{*}} \int_{0}^{+\infty} C e^{t A} B d t=\frac{\beta x^{*}}{\mu_{v}+u \beta x^{*}} C\left(-A^{-1}\right) B .
$$

This proves

$$
\mathcal{R}_{0}=\frac{\beta x^{*}}{\mu_{v}+u \beta x^{*}} C\left(-A^{-1}\right) B .
$$

With our definition we have $C\left(-A^{-1}\right) B=\gamma_{k} e_{e n d}(k)^{T}\left(-A^{-1}\right) e_{1}(k)$ which is simply the entry of the last row, first column of $-A^{-1}$ multiplied by $\gamma_{k}$. Finally

$$
\mathcal{R}_{0}=\frac{\beta x^{*}}{\mu_{v}+u \beta x^{*}} \frac{\gamma_{1} \cdots \gamma_{k}}{\alpha_{1} \cdots \alpha_{k}} .
$$

We also define a threshold $\mathcal{T}_{0}$ by

$$
\mathcal{T}_{0}=\frac{\beta\left[\frac{\gamma_{1} \cdots \gamma_{k}}{\alpha_{1} \cdots \alpha_{k}}-u\right] x^{*}}{\mu_{v}} .
$$

We call $\mathcal{T}_{0}$ a threshold since $\mathcal{T}_{0} \leq 1$ is equivalent to $\mathcal{R}_{0} \leq 1$.

The system has two nonnegative equilibria. The first, called the parasite free equilibrium $(\mathrm{PFE})$, is $\left(x^{*}, 0 \cdots, 0\right)$. The second is called the endemic equilibrium $(\mathrm{EE})$ and is denoted by $(\bar{x}, \bar{y}, \bar{v})$.

We have necessarily $\bar{y}=\beta \bar{x} \bar{v}\left(-A^{-1}\right) e_{1}$ and

$$
\mu_{v} \bar{v}+u \beta \bar{x} \bar{v}=\gamma_{k} \beta \bar{x} \bar{v} e_{e n d}^{T}\left(-A^{-1}\right) e_{1} .
$$

If $\bar{v} \neq 0$ we deduce

$$
\bar{x}=\frac{\mu_{v}}{\beta\left[\gamma_{k} e_{\text {end }}^{T}\left(-A^{-1}\right) e_{1}-u\right]}=\frac{x^{*}}{\mathcal{T}_{0}} .
$$

With this expression we get $\bar{v}=\frac{\varphi(\bar{x})}{\beta \bar{x}}$. Hence, with the hypothesis $(13), \bar{x}$ and $\bar{v}$ are positive iff $\mathcal{T}_{0}>1$ or equivalently iff $\mathcal{R}_{0}>1$. Now $\bar{y}=\varphi(\bar{x})\left(-A^{-1}\right) e_{1}$. In other words $\bar{y}$ is the first column of $\left(-A^{-1}\right)$ multiplied by $\varphi(\bar{x})$. The first column of $\left(-A^{-1}\right)$ is a positive vector, hence $v$ is in the positive orthant, classically denoted by $\bar{v} \gg 0$.

To summarize the endemic equilibrium is in the positive orthant iff $\mathcal{R}_{0}>1$ and it is given by

$$
\left\{\begin{array}{l}
\bar{x}=\frac{\mu_{v}}{\beta\left[\frac{\gamma_{1} \cdots \gamma_{k}}{\alpha_{1} \cdots \alpha_{k}}-u\right]}=\frac{x^{*}}{\mathcal{T}_{0}}<x^{*}, \\
\bar{y}=\varphi(\bar{x})(-A)^{-1} e_{1}, \\
\bar{v}=\frac{\varphi(\bar{x})}{\beta \bar{x}} .
\end{array}\right.
$$




\subsection{Stability analysis}

We give the main result of this section.

Theorem 1 We consider the system (14) with the hypothesis on $\varphi$ (13) satisfied. The basic reproduction ratio of the system is given by (17).

(1) The system (14) is globally asymptotically stable on $\mathbb{R}_{+}^{k+2}$ at the parasite free equilibrium (PFE) $\left(x^{*}, 0, \cdots, 0\right)$ if and only if $\mathcal{R}_{0} \leq 1$.

(2) If $\mathcal{R}_{0}>1$ then the PFE is unstable and there exists a unique endemic equilibrium (EE) in the positive orthant, $(\bar{x}, \bar{y}, \bar{v}) \gg 0$ given by (19)

(3) If $\mathcal{R}_{0}>1$, and if

$$
u \beta \varphi(\bar{x}) \leq-\mu_{v} \max _{x \in\left[0, x^{*}\right]}\left(\varphi^{\prime}(x)\right),
$$

then the endemic equilibrium is globally asymptotically stable on the nonnegative orthant, excepted for initial conditions on the $x$-axis.

Remark 1 If $\varphi$ increases on some part of its domain, the relation (20) is never satisfied. If this happens, it may lead to limit cycle for this model as in [40].

Remark 2 When $u=0$ and $\varphi(x)=\Lambda-\mu_{x} x$ the sufficient condition (20) is automatically satisfied. This is the case of numerous models of the literature. See for example the general model (1) of [15] or the model in [21].

Proof. We need some dissipativity properties of system (14). In a first step we show that there exists in the nonnegative orthant $\mathbb{R}_{+}^{k+2}$ a forward invariant compact absorbing neighborhood $\Omega$ of the PFE $\left(x^{*}, 0, \cdots, 0\right)$. An absorbing set $D$ is a neighborhood of the PFE such that the trajectory of the system starting from any initial condition enters and remains in $D$ for a sufficiently large time $T$. The entrance time depends on the initial condition. If the initial conditions are contained in a compact set $F$ then there exits a uniform $T$ for $F$. A system is point dissipative if there exists a compact absorbing set. The above definition coincides with dissipativity given by [33]. See also [34] for the different notions of dissipativity.

Let $\varepsilon \geq 0$ be a given nonnegative real. Thanks to the hypothesis $(13)$ on $\varphi$, for any initial condition in the nonnegative orthant there exists a time $T>0$ such that and for $t \geq T$ we have $x(t) \leq x^{*}+\varepsilon$. Let $M_{\varphi}$ be the maximum of the function $\varphi(x)$ on $\mathbb{R}_{+}$, and let $\rho$ be a positive real such that $\alpha_{1} \rho>M_{\varphi}+\varepsilon$. 
We claim that the set $\mathcal{D}_{\varepsilon}$ defined by

$$
\begin{aligned}
& \mathcal{D}_{\varepsilon}=\left\{(x, y, v) \in \mathbb{R}_{+}^{k+2} \mid x \leq x^{*}+\varepsilon, x+y_{1} \leq \rho+x^{*}+\varepsilon\right. \\
& \text { and for } \left.i=2 \cdots k, y_{i} \leq \frac{\gamma_{2} \cdots \gamma_{i-1}}{\alpha_{2} \cdots \alpha_{i}}\left(\rho+x^{*}+i \varepsilon\right), v \leq \frac{\gamma_{1} \cdots \gamma_{k}}{\alpha_{2} \cdots \alpha_{k} \mu_{v}}\left(\rho+x^{*}+k \varepsilon\right)\right\}
\end{aligned}
$$

is a forward invariant compact absorbing set for the system for $\varepsilon>0$, and that the set $\mathcal{D}_{0}(\varepsilon=0)$ is a forward invariant compact set.

The set $\mathcal{D}_{\varepsilon}$ is the intersection of half-spaces defined by some hyperplanes. To prove the positive invariance of a set, it is sufficient to prove that the vector field associated to the system is tangent or pointing toward the interior of the set on the boundary of this set [35. This is immediate for the faces of the nonnegative orthant and for the half-space defined by $\mathcal{D}_{\varepsilon, 1}=\{(x, y, v) \mid$ $\left.x \leq x^{*}+\varepsilon\right\}$. From the properties of $\varphi$ this set is also clearly absorbing. We define $\mathcal{D}_{\varepsilon, 2}=\left\{(x, y, v) \in \mathcal{D}_{\varepsilon, 1} \mid x+y_{1} \leq \rho+x^{*}+2 \varepsilon\right\}$. We have just to look at the boundary of $\mathcal{D}_{\varepsilon, 2}$ contained in $\mathcal{D}_{\varepsilon, 1}$. On this part of the boundary we have $y_{1} \geq \rho$. So on this boundary we have $\dot{x}+\dot{y}_{1} \leq M_{\varphi}-\alpha_{1} y_{1}<\varepsilon$. This shows that the vector points toward the interior of $\mathcal{D}_{\varepsilon, 2}$, hence $\mathcal{D}_{\varepsilon, 2}$ is positively invariant. The inequality $\dot{x}+\dot{y}_{1}<\varepsilon$ proves that $\mathcal{D}_{\varepsilon, 2}$ is absorbing in $\mathcal{D}_{\varepsilon, 1}$. A finite induction process, with similar arguments, ends the proof for $\mathcal{D}_{\varepsilon}$.

In a second step we will prove that, if $\mathcal{R}_{0} \leq 1$ then the PFE is globally asymptotically stable on the compact forward invariant set $\mathcal{D}_{0}$. It is well known that if $\mathcal{R}_{0}>1$ then the PFE is unstable [25|24]. Thus the condition $\mathcal{R}_{0} \leq 1$ is necessary.

To prove the sufficiency we consider the following Liapunov function on the positive orthant.

$$
V_{P F E}(y, v)=b^{T} y+v
$$

where the column vector $b=\left[b_{1} ; b_{2} ; \cdots ; b_{k}\right]$ is the transpose of the last row of $-A^{-1}$ multiplied by $\gamma_{k}$. In other words $b=\gamma_{k}\left(-A^{-T}\right) e_{e n d}$.

We also define for further reference $a=b_{1}-u$. If we use (19) we obtain for the parameter $a$ the equivalent relation

$$
a=b_{1}-u=\frac{\mu_{v}}{\beta \bar{x}}=\left[\frac{\gamma_{1} \cdots \gamma_{k}}{\alpha_{1} \cdots \alpha_{k}}-u\right]
$$


If we compute the derivative of $V_{P F E}$ along the trajectories of 14 we get

$$
\begin{aligned}
\dot{V}_{P F E} & =b^{T} \dot{y}+\dot{v} \\
& =\gamma_{k} e_{\text {end }}^{T}\left(-A^{-1}\right) A y+\gamma_{k} e_{e n d}^{T}\left(-A^{-1}\right) \beta x v e_{1}+\dot{v} \\
& =-\gamma_{k} y_{k}+\beta x v b_{1}+\gamma_{k} y_{k}-\mu_{v} v-u \beta x v \\
& =v\left[\left(b_{1}-u\right) \beta x-\mu_{v}\right]=v\left[a \beta x-\mu_{v}\right] \\
& =v\left[\frac{\mu_{v} x}{\bar{x}}-\mu_{v}\right] \\
& =\frac{\mu_{v}}{\bar{x}}(x-\bar{x}) v \\
& =\beta\left[\frac{\gamma_{1} \cdots \gamma_{k}}{\alpha_{1} \cdots \alpha_{k}}-u\right](x-\bar{x}) v .
\end{aligned}
$$

If $\mathcal{R}_{0} \leq 1$, or equivalently $\mathcal{T}_{0} \leq 1$, we distinguish two cases :

(1) On one hand if $\left(\frac{\gamma_{1} \cdots \gamma_{k}}{\alpha_{1} \cdots \alpha_{k}}-u\right)<0$ then $\bar{x}<0$ and all the other quantities are nonnegative in the expression of $\dot{V}$. Therefore $\dot{V} \leq 0$.

(2) On the other hand if $\left(\frac{\gamma_{1} \cdots \gamma_{k}}{\alpha_{1} \cdots \alpha_{k}}-u\right) \geq 0$, then from $\mathcal{T}_{0} \leq 1$ and since we are in $\mathcal{D}_{0}$ we deduce that $0 \leq x \leq x^{*} \leq \bar{x}$, and hence it follows that $\dot{V} \leq 0$.

In both cases $\dot{V} \leq 0$. It is easy to see that the maximum invariant set in $\left\{(x, y, v) \in \mathcal{D}_{0} \mid \dot{V}=0\right\}$ is reduced to the PFE. Therefore the global asymptotic stability of the PFE on the compact positively invariant set $\mathcal{D}_{0}$ follows from ([36], Theorem 3.7.11, page 346). Now, We will prove the global asymptotic stability on the orthant $\mathbb{R}_{+}^{k+2}$. It is sufficient to prove that any forward trajectory converges to the PFE. Since $\mathcal{D}_{1}$ ( i.e., $\mathcal{D}_{\varepsilon}$ for $\varepsilon=1$ ) is a forward compact absorbing set any trajectory enters $\mathcal{D}_{1}$. If a trajectory enters the interior of $\mathcal{D}_{0}$ we have already proved that it converges toward the PFE. Now assume that a trajectory, in $\mathcal{D}_{1}$ stays in $\mathcal{D}_{1} \cap\left\{x^{*} \leq x \leq x^{*}+1\right\}$. Consider the Liapunov function $W(x)=\frac{1}{2}\left(x-x^{*}\right)^{2}$ on this trajectory. Thanks to the hypothesis (13) on $\varphi$ and the hypothesis on the trajectory we have $\dot{W}=\left(x-x^{*}\right) \varphi(x)-\left(x-x^{*}\right) \beta x v \leq 0$ on any point of the trajectory in $\mathcal{D}_{1}$. By LaSalle's invariance principle [37] it follows from $\dot{W} \leq 0$ that the trajectory converges toward the PFE since the PFE is the largest invariant set contained in $\left\{(x, y, v) \in D_{1}, x^{*} \leq x \leq x^{*}+1 \mid \dot{W}=0\right\}$. This ends the proof of the GAS of the PFE.

Now we assume that $\mathcal{R}_{0}>1$. The equilibrium $(\bar{x}, \bar{y}, \bar{v})$ of the system, different from the PFE, is given by (19) and it belongs to the positive orthant since $\mathcal{R}_{0}>1$. 
We will now prove that (20) is a sufficient condition for the GAS of the EE. To this end we define the following Liapunov function on the positive orthant.

$$
V_{E E}(x, y, v)=a(x-\bar{x} \ln x)+\sum_{i=1}^{k} b_{i}\left(y_{i}-\bar{y}_{i} \ln y_{i}\right)+(v-\bar{v} \ln v),
$$

where the column vector $b$ and the coefficient $a$ have been previously defined by the relation (22). Since $\mathcal{R}_{0}>1$ we deduce $a>0$, hence the coefficients of $V_{E E}$ are positive. In this case this function has a unique minimum, the EE, in the positive orthant.

This function has a linear part $L_{E E}(x, y, v)=a x+\sum_{i=1}^{k} b_{i} y_{i}+v$. This linear part can be expressed as

$$
L_{E E}(x, y, v)=a x+b^{T} y+v=a x+\gamma_{k} e_{e n d}\left(-A^{-1}\right) y+v .
$$

If we compute the derivative $\dot{L}_{E E}$ of $L_{E E}$ along the trajectories of $(14)$, considering the definition of $b$ and the relation $a+u=b_{1}$, we get

$$
\begin{aligned}
\dot{L}_{E E}(x, y, v) & =a \dot{x}+\gamma_{k} e_{e n d}\left(-A^{-1}\right) \dot{y}+\dot{v} \\
& =a \dot{x}+\gamma_{k} e_{\text {end }}\left(-A^{-1}\right) A y+\beta x v e_{\text {end }}\left(-A^{-1}\right) B+\dot{v} \\
& =a \dot{x}-\gamma_{k} e_{\text {end }} y+\beta x v e_{\text {end }}\left(-A^{-1}\right) e_{1}+\dot{v} \\
& =a \varphi(x)-a \beta x v-\gamma_{k} y_{k}+b_{1} \beta x v+\gamma_{k} y_{k}-\mu_{v} v-u \beta x v \\
& =a \varphi(x)-\mu_{v} v .
\end{aligned}
$$

If we collect in $\dot{V}_{E E}$ the terms in $v$ we obtain $\left(a \beta \bar{x}-\mu_{v}\right) v$. From 22 the terms in $v$ cancel. With these simplifications we can now express $\dot{V}_{E E}$

$$
\begin{gathered}
\dot{V}_{E E}=a \varphi(x)\left(1-\frac{\bar{x}}{x}\right)-b_{1} \beta \bar{y}_{1} \frac{x v}{y_{1}}-\sum_{i=2}^{k} b_{i} \gamma_{i-1} y_{i-1} \frac{\bar{y}_{i}}{y_{i}}+ \\
+\sum_{i=1}^{k} b_{i} \alpha_{i} \bar{y}_{i}-\gamma_{k} y_{k} \frac{\bar{v}}{v}+u \beta \bar{v} x+\mu_{v} \bar{v} .
\end{gathered}
$$

This can also be written

$$
\begin{aligned}
\dot{V}_{E E} & =a \varphi(x)\left(1-\frac{\bar{x}}{x}\right)-b_{1} \beta \bar{x} \bar{v} \frac{x}{\bar{x}} \frac{v}{\bar{v}} \frac{\bar{y}_{1}}{y_{1}}-\sum_{i=2}^{k} b_{i} \gamma_{i-1} \bar{y}_{i-1} \frac{y_{i-1}}{\bar{y}_{i-1}} \frac{\bar{y}_{i}}{y_{i}}+ \\
& +\sum_{i=1}^{k} b_{i} \alpha_{i} \bar{y}_{i}-\gamma_{k} \bar{y}_{k} \frac{y_{k}}{\bar{y}_{k}} \frac{\bar{v}}{v}+u \beta \bar{x} \bar{v} \frac{x}{\bar{x}}+\mu_{v} \bar{v} .
\end{aligned}
$$

We now compare some coefficients appearing in this formula. We have $\varphi(\bar{x})=$ $\beta \bar{x} \bar{v}$. Using the fact that $\bar{y}$ is the first column of $-A^{-1}$ multiplied by $\varphi(\bar{x})$, the column vector $b$ is the transpose of the last row of the matrix $-A^{-1}$ multiplied 
by $\gamma_{k}$ and accordingly to the relation $(15)$, we have:

$$
\begin{aligned}
b_{i} \gamma_{i-1} \bar{y}_{i-1} & =\gamma_{k}\left(-A^{-1}\right)(k, i) \gamma_{i-1} \varphi(\bar{x})\left(-A^{-1}\right)(i-1,1) \\
& =\varphi(\bar{x}) \gamma_{k} \frac{\gamma_{i} \cdots \gamma_{k-1}}{\alpha_{i} \ldots \alpha_{k-1}} \frac{1}{\alpha_{k}} \gamma_{i-1} \frac{\gamma_{1} \cdots \gamma_{i-2}}{\alpha_{1} \cdots \alpha_{i-2}} \frac{1}{\alpha_{i-1}} \\
& =\frac{\gamma_{1} \cdots \gamma_{k}}{\alpha_{1} \cdots \gamma_{k}} \varphi(\bar{x})=b_{1} \varphi(\bar{x})=b_{1} \beta \bar{x} \bar{v} .
\end{aligned}
$$

In the same way

$$
\begin{aligned}
b_{i} \alpha_{i} \bar{y}_{i} & =\gamma_{k}\left(-A^{-1}\right)(k, i) \alpha_{i} \varphi(\bar{x})\left(-A^{-1}\right)(i, 1) \\
& =\varphi(\bar{x}) \gamma_{k} \frac{\gamma_{i} \cdots \gamma_{k-1}}{\alpha_{i} \cdots \alpha_{k-1}} \frac{1}{\alpha_{k}} \alpha_{i} \frac{\gamma_{1} \cdots \gamma_{i-1}}{\alpha_{1} \cdots \alpha_{i-1}} \frac{1}{\alpha_{i}}=\frac{\gamma_{1} \cdots \gamma_{k}}{\alpha_{1} \cdots \gamma_{k}} \varphi(\bar{x})=b_{1} \varphi(\bar{x}) .
\end{aligned}
$$

and $\gamma_{k} \bar{y}_{k}=\gamma_{k}\left(-A^{-1}\right)(k, i) \varphi(\bar{x})=\varphi(\bar{x}) \gamma_{k} \frac{\gamma_{1} \cdots \gamma_{k-1}}{\alpha_{1} \ldots \alpha_{k-1}} \frac{1}{\alpha_{k}}=b_{1} \varphi(\bar{x})$.

Thanks to 22 we also have $\mu_{v} \bar{v}=a \beta \bar{x} \bar{v}=a \varphi(\bar{x})$.

Using all these relations between the coefficients we get for $\dot{V}_{E E}$

$$
\begin{gathered}
\dot{V}_{E E}=a \varphi(x)\left(1-\frac{\bar{x}}{x}\right)+u \varphi(\bar{x}) \frac{x}{\bar{x}}+a \varphi(\bar{x})+ \\
b_{1} \varphi(\bar{x})\left[k-\frac{x}{\bar{x}} \frac{v}{\bar{v}} \frac{\bar{y}_{1}}{y_{1}}-\sum_{i=2}^{k} \frac{y_{i-1}}{\bar{y}_{i-1}} \frac{\bar{y}_{i}}{y_{i}}-\frac{y_{k}}{\bar{y}_{k}} \frac{\bar{v}}{v}\right] .
\end{gathered}
$$

Adding $2-\frac{\bar{x}}{x}$ in the expression between brackets and subtracting the same expression outside the brackets, and using $u=b_{1}-a$ we obtain

$$
\begin{gathered}
\dot{V}_{E E}=a \varphi(x)\left(1-\frac{\bar{x}}{x}\right)+b_{1} \varphi(\bar{x})\left(\frac{\bar{x}}{x}+\frac{x}{\bar{x}}-2\right)+a \varphi(\bar{x})\left(1-\frac{x}{\bar{x}}\right)+ \\
b_{1} \varphi(\bar{x})\left[k+2-\frac{\bar{x}}{x}-\frac{x}{\bar{x}} \frac{v}{\bar{v}} \frac{\bar{y}_{1}}{y_{1}}-\sum_{i=2}^{k} \frac{y_{i-1}}{\bar{y}_{i-1}} \frac{\bar{y}_{i}}{y_{i}}-\frac{y_{k}}{\bar{y}_{k}} \frac{\bar{v}}{\bar{v}}\right] .
\end{gathered}
$$

This can also be written

$$
\begin{gathered}
\dot{V}_{E E}=\frac{x-\bar{x}}{x \bar{x}}\left(a \bar{x} \varphi(x)-a x \varphi(\bar{x})+b_{1} \varphi(\bar{x})(x-\bar{x})\right)+ \\
b_{1} \varphi(\bar{x})\left[k+2-\frac{\bar{x}}{x}-\frac{x}{\bar{x}} \frac{v}{\bar{v}} \frac{\bar{y}_{1}}{y_{1}}-\sum_{i=2}^{k} \frac{y_{i-1}}{\bar{y}_{i-1}} \frac{\bar{y}_{i}}{y_{i}}-\frac{y_{k}}{\bar{y}_{k}} \frac{\bar{v}}{v}\right] .
\end{gathered}
$$

Now we will use the fact that there exists $\xi$ in the open interval $] x, \bar{x}$ [ such that $\varphi(x)=\varphi(\bar{x})+(x-\bar{x}) \varphi^{\prime}(\xi)$. Replacing in the preceding expression gives

$$
\begin{aligned}
& \dot{V}_{E E}=\frac{(x-\bar{x})^{2}}{x \bar{x}}\left(-a \varphi(\bar{x})+a \bar{x} \varphi^{\prime}(\xi)+b_{1} \varphi(\bar{x})\right)+ \\
& b_{1} \varphi(\bar{x})\left[k+2-\frac{\bar{x}}{x}-\frac{x}{\bar{x}} \frac{v}{\bar{v}} \frac{\bar{y}_{1}}{y_{1}}-\sum_{i=2}^{k} \frac{y_{i-1}}{\bar{y}_{i-1}} \frac{\bar{y}_{i}}{y_{i}}-\frac{y_{k}}{\bar{y}_{k}} \frac{\bar{v}}{v}\right] .
\end{aligned}
$$


Finally since $a-b_{1}=u$,

$$
\begin{aligned}
\dot{V}_{E E} & =\frac{(x-\bar{x})^{2}}{x \bar{x}}\left(u \varphi(\bar{x})+a \bar{x} \varphi^{\prime}(\xi)\right)+ \\
& b_{1} \varphi(\bar{x})\left[k+2-\frac{\bar{x}}{x}-\frac{x}{\bar{x}} \frac{v}{\bar{v}} \frac{\bar{y}_{1}}{y_{1}}-\sum_{i=2}^{k} \frac{y_{i-1}}{\bar{y}_{i-1}} \frac{\bar{y}_{i}}{y_{i}}-\frac{y_{k}}{\bar{y}_{k}} \frac{\bar{v}}{\bar{v}}\right] .
\end{aligned}
$$

On the one hand, the inequality between the arithmetical mean and the geometrical mean implies that the term between brackets in the last expression of $\dot{V}$ is non positive and it vanishes if and only if $(x, y, v)=(\bar{x}, \bar{y}, \bar{v})$. On the other hand, thanks to the assumption $u \beta \varphi(\bar{x}) \leq-\mu_{v} \max _{x \in\left[0, x^{*}\right]} \varphi^{\prime}(x)$, and to the relation $a \bar{x}=\frac{\mu_{v}}{\beta}$, we have

$$
u \varphi(\bar{x})+a \bar{x} \varphi^{\prime}(\xi) \leq 0
$$

Therefore $\dot{V}$ is negative excepted at the EE for the system 14 .

Since $V_{E E}$ is a proper function on the positive orthant, this proves the GAS of the EE on the positive orthant for the system (14).

The vector field associated to the system (14) points toward the interior of the orthant on all the faces except on the $x$-axis where it is tangent. Therefore the basin of attraction of the EE is the orthant excepted the one-dimensional face contained in the $x$-axis of the orthant, which is the stable manifold of the PFE. This ends the proof of the theorem.

\section{Stability analysis for the complete system}

Using the computations of the preceding section we can study the stability of the complete system (4) or equivalently of system (12) with $A, B$ and $C$ defined in Subsection 3.1.

\subsection{Equilibria and $\mathcal{R}_{0}$}

The one chain system (14) can be viewed as a particular case of system (12) with all the $\pi_{j}=0$ but one which satisfies for index $i, \pi_{i}=1$. Hence we define

$$
\mathcal{R}_{0}^{i}=\frac{\beta x^{*}}{\mu_{v}+u \beta x^{*}} \frac{\gamma_{1, i} \cdots \gamma_{k_{i}, i}}{\alpha_{1, i} \cdots \alpha_{k_{i}, i}} .
$$

We recall that $n=\sum_{i=1}^{q} k_{i}$. We decompose the vector $y$ of $\mathbb{R}^{n}$ in $k$ blocks of respective length $k_{1}, k_{2}, \ldots, k_{q}: y=\left[y_{1} ; \ldots ; y_{q}\right]$, with $y_{i}=\left[y_{1, i} ; \ldots ; y_{k_{i}, i}\right]$. 
In the same way we write $B=\left[B_{1} ; \ldots ; B_{q}\right]$, and similarly for the row vector $1 \times n$ we write $C=\left[C_{1}, \ldots, C_{q}\right]$. From Subsection 3.1, we have $B_{i}=\pi_{i} e_{1}\left(k_{i}\right)$ and $C_{i}=\gamma_{k_{i}, i} e_{\text {end }}\left(k_{i}\right)^{T}$.

An analogous computation as in Section 4 allows to obtain the following formula which is analogous to the formula (16):

$$
\mathcal{R}_{0}^{i}=\frac{\beta x^{*}}{\mu_{v}+u \beta x^{*}} \frac{1}{\pi_{i}} C_{i}\left(-A_{i}^{-1}\right) B_{i} .
$$

The same reasoning as in Subsection 4.2 leads to evaluate the output of $\dot{y}=$ $A y+w B$ for the Dirac input $w=\frac{\beta x^{*}}{\mu_{v}+u \beta x^{*}}$. It is clear then that

$$
\mathcal{R}_{0}=\frac{\beta x^{*}}{\mu_{v}+u \beta x^{*}} C\left(-A^{-1}\right) B
$$

We define also

$$
\mathcal{T}_{0}=\frac{\beta x^{*}\left[C\left(-A^{-1}\right) B-u\right]}{\mu_{v}} .
$$

Now, due to the block structure of the matrices, we have

$$
C\left(-A^{-1}\right) B=\sum_{i=1}^{q} C_{i}\left(-A_{i}^{-1}\right) B_{i}
$$

Hence it follows

$$
\mathcal{R}_{0}=\sum_{i=1}^{q} \pi_{i} \mathcal{R}_{0}^{i}
$$

There exist two equilibria, the PFE $\left[x^{*} ; 0 ; \cdots ; 0\right]$ and an endemic equilibrium $[\bar{x} ; \bar{y} ; \bar{v}]$ given by

$$
\left\{\begin{array}{l}
\bar{x}=\frac{\mu_{v}}{\beta\left[C\left(-A^{-1}\right) B-u\right]}=\frac{x^{*}}{\mathcal{T}_{0}}, \\
\bar{y}=\varphi(\bar{x})(-A)^{-1} B \\
\bar{v}=\frac{\varphi(\bar{x})}{\beta \bar{x}} .
\end{array}\right.
$$

The blocks for $\bar{y}$ are immediate

$$
\bar{y}_{i}=\varphi(\bar{x})\left(-A_{i}^{-1}\right) B_{i}=\pi_{i} \varphi(\bar{x})\left(-A_{i}^{-1}\right) e_{1}\left(k_{i}\right) .
$$

The endemic equilibrium belongs to the positive orthant iff $\mathcal{R}_{0}>1$.

\subsection{Main result}

The main result is identical to the one chain result: 
Theorem 2 We consider the system (12) with the hypothesis (13) satisfied. The basic reproduction ratio of the system is given by (26).

(1) The system (12) is globally asymptotically stable on $\mathbb{R}_{+}^{n+2}$ at the parasite free equilibrium (PFE) $\left(x^{*}, 0, \ldots, 0\right)$ if and only if $\mathcal{R}_{0} \leq 1$.

(2) If $\mathcal{R}_{0}>1$ then the PFE is unstable, and there exists a unique endemic equilibrium (EE) in the positive orthant, $(\bar{x}, \bar{y}, \bar{v}) \gg 0$.

(3) If $\mathcal{R}_{0}>1$, and if

$$
u \beta \varphi(\bar{x}) \leq-\mu_{v} \max _{x \in\left[0, x^{*}\right]}\left(\varphi^{\prime}(x)\right)
$$

then the endemic equilibrium is globally asymptotically stable on the nonnegative orthant, excepted for initial conditions on the nonnegative $x$-axis.

Proof. In a first step we will prove that if $\mathcal{R}_{0} \leq 1$ all the trajectories of $(12)$ are forward bounded. We consider the Liapunov function on the nonnegative orthant

$$
V_{P F E}=b^{T} y+v
$$

Where $b=\left(-A^{-T}\right) C^{T}$. We shall prove that the derivative $\dot{V}_{P F E}$ of $V_{P F E}$ along the trajectories of 12 is nonpositive if $x \leq x^{*}$. We have

$$
\begin{aligned}
\dot{V}_{P F E} & =C\left(-A^{-1}\right) A y+\beta x v C\left(-A^{-1}\right) B+\dot{v} \\
& =-C y+\beta x v C\left(-A^{-1}\right) B+C y-\mu_{v} v-u \beta x v \\
& =\beta v\left[C\left(-A^{-1}\right) B-u\right]\left(x-\frac{\mu_{v}}{\beta\left[C\left(-A^{-1}\right) B-u\right]}\right) \\
& =\beta v\left[C\left(-A^{-1}\right) B-u\right](x-\bar{x}) .
\end{aligned}
$$

In case $\left[C\left(-A^{-1}\right) B-u\right]=0, \dot{V}_{P F E}$ is simply $\dot{V}_{P F E}=-\mu_{v} v$.

If $\left[C\left(-A^{-1}\right) B-u\right]<0$, then $\bar{x}<0$, and so $\dot{V}_{P F E} \leq 0$. If $\left[C\left(-A^{-1}\right) B-u\right]>0$, then $\bar{x}>0$ but we have $x^{*} \leq \bar{x}$ since $\mathcal{T}_{0} \leq 1$, so $\dot{V}_{P F E} \leq 0$ if $x \leq x^{*}$.

As a consequence if we denote $H_{x^{*}}$ the set in the nonnegative orthant defined by $H_{x^{*}}=\left\{(x, y, v) \in \mathbb{R}_{+}^{n+2} \mid x \leq x^{*}\right\}$ and, for $\kappa \in \mathbb{R}_{+}, \Omega_{1, \kappa}$ the set

$$
\Omega_{1, k}=V_{P F E}^{-1}([0, \kappa]) \cap H_{x^{*}} .
$$

Then, the set $H_{x^{*}}$ is a forward invariant set and for any $\kappa \in \mathbb{R}_{+}$, the set $\Omega_{1, \kappa}$ is a compact forward invariant set for (12).

Now we define another function $W$ on the nonnegative orthant by $W(x, y, v)=$ $C\left(-A^{-1}\right) B x+V_{P F E}(y, v)$. We have immediately

$$
\dot{W}=C\left(-A^{-1}\right) B \varphi(x)-\mu_{v} v-u \beta x v .
$$


The hypothesis 13 on $\varphi(x)$ implies that $\dot{W} \leq 0$ if $x \geq x^{*}$.

We define the set $\Omega_{2, \kappa}$ contained in the nonnegative orthant by

$$
\Omega_{2, \kappa}=W_{P F E}^{-1}([0, \kappa]) \cap\left\{(x, y, v) \in \mathbb{R}^{n+2} \mid x \geq x^{*}\right\} .
$$

Since $V_{P F E} \leq W$ on the nonnegative orthant we conclude that the set $\Omega_{\kappa}$ defined by

$$
\Omega_{\kappa}=\Omega_{1, \kappa} \cup \Omega_{2, \kappa}
$$

is a compact forward invariant set for 12 . For, a trajectory in $\Omega_{2, \kappa}$ can leave the set only by the boundary $x=x^{*}$ in which case it enters $\Omega_{1, \kappa}$ which is forward invariant. We have then proved that all the trajectories of $(12)$ are bounded if $\mathcal{R}_{0} \leq 1$ since any initial condition $\left(x_{0}, y_{0}, v_{0}\right) \in \mathbb{R}_{+}^{n+2}$ is contained in one $\Omega_{\kappa}$.

The second step is to prove the global asymptotic stability (GAS) of the PFE.

For an initial condition in the compact set $\Omega_{1, \kappa}$ we use the Liapunov function $V_{P F E}$. The conclusion follows from ([36], Theorem 3.7.11, page 346), since the largest invariant set contained in $\left\{\dot{V}_{P F E}=0\right\}$ is $\left\{\left(x^{*}, 0,0\right)\right\}$.

For an initial condition in $\Omega_{2, \kappa}$ either the trajectory enters $\Omega_{1, \kappa}$, in which case the preceding argument applies, or the forward trajectory stays in $\Omega_{2, \kappa}$. In this case the function $W$ is decreasing on this trajectory. The omega limit set of this trajectory is included in $\dot{W}=0$. But $\dot{W}=0$ is reduced to $\left\{\left(x^{*}, 0,0\right)\right\}$ since $C\left(-A^{-1}\right) B>0$ and $\varphi$ satisfies the hypothesis $(13)$. This ends the proof of the global stability of the PFE.

To prove the global stability for the EE we consider the following Liapunov function

$$
V_{E E}(x, y, v)=a(x-\bar{x} \ln x)+\sum_{i=1}^{k} b_{i}\left(y_{i}-\bar{y}_{i} \ln y_{i}\right)+(v-\bar{v} \ln v)
$$

with $a$ defined by $a=C\left(-A^{-1}\right) B-u=\frac{\mu_{v}}{\beta \bar{x}}$ and $b=\left(-A^{-T}\right) C^{T}$ as before. The vector $b$ can be written in $q$ blocks of size $k_{1} \times 1, \ldots, k_{q} \times 1: b=\left[b_{1} ; b_{2} ; \ldots: b_{q}\right]$. We denote by $b_{j, i}$ the $j$-th component of the vector block $b_{i}$. 
As in the case of the one chain system, the terms in $y_{i}, v$ and $x v$, appearing in the expression of $\dot{V}_{E E}$ cancel. Then we have

$$
\begin{aligned}
\dot{V}_{E E} & =a \varphi(x)\left(1-\frac{\bar{x}}{x}\right) \\
& +\sum_{i=1}^{q}\left[-\pi_{i} b_{1, i} \beta \bar{x} \bar{v} \frac{y_{1, i}}{\bar{y}_{1, i}} \frac{x}{\bar{x}} \frac{v}{\bar{v}}-\sum_{j=2}^{k_{i}} b_{j, i} \gamma_{j-1, i} \bar{y}_{j-1, i} \frac{y_{j-1, i}}{\bar{y}_{j-1, i}} \frac{\bar{y}_{j, i}}{y_{j, i}}\right. \\
& \left.+\sum_{j=1}^{k_{i}} b_{j, i} \alpha_{j, i} \bar{y}_{j, i}-\gamma_{k_{i}, i} \bar{y}_{k_{i}, i} \frac{y_{k_{i}, i}}{\bar{y}_{k_{i}, i}} \bar{v}\right]+\mu_{v} \bar{v}+u \beta \bar{x} \bar{v} \frac{x}{\bar{x}} .
\end{aligned}
$$

Each diagonal block $A_{i}$ of the matrix $A$ has the same structure as the matrix considered in the one chain case. The relation (28) for the blocks $\bar{y}_{i}$ of $y$ allows to deduce, as in the proof of the theorem for the one chain case, the following relations between the coefficients

$$
b_{j, i} \gamma_{j-1, i} \bar{y}_{j-1, i}=\pi_{i} b_{1, i} \varphi(\bar{x})=b_{j, i} \alpha_{i, j} \bar{y}_{j, i}=\gamma_{k_{i}, i} \bar{y}_{k_{i}, i},
$$

and also $\mu_{v} \bar{v}=a \beta \bar{x} \bar{v}=a \varphi(\bar{x})$. Hence

$$
\begin{aligned}
\dot{V}_{E E} & =a \varphi(x)\left(1-\frac{\bar{x}}{x}\right)+a \varphi(\bar{x})+u \varphi(\bar{x}) \frac{x}{\bar{x}} \\
& +\varphi(\bar{x}) \sum_{i=1}^{q} \pi_{i} b_{1, i}\left[k_{i}-\frac{y_{1, i}}{\bar{y}_{1, i}} \frac{x}{\bar{x}} \frac{v}{\bar{v}}-\sum_{j=2}^{k_{i}} \frac{y_{j-1, i}}{\bar{y}_{j-1, i}} \frac{\bar{y}_{j, i}}{y_{j, i}}-\frac{y_{k_{i}, i}}{\bar{y}_{k_{i}, i}} \frac{\bar{v}}{v}\right] .
\end{aligned}
$$

We recall that $a+u=C\left(-A^{-1}\right) B=\sum_{i=1}^{q} C_{i}\left(-A_{i}^{-1}\right) B_{i}=\sum_{i=1}^{q} \pi_{i} b_{1, i}$. So if we add, between the brackets of the preceding expression of $\dot{V}_{E E}$, the term $2-\frac{\bar{x}}{x}$ we must subtract $(a+u) \varphi(\bar{x})\left(\frac{\bar{x}}{x}-2\right)$ outside the sum, hence

$$
\begin{aligned}
\dot{V}_{E E} & =a \varphi(x)\left(1-\frac{\bar{x}}{x}\right)+a \varphi(\bar{x})\left(1-\frac{x}{\bar{x}}\right)+(a+u) \varphi(\bar{x})\left(\frac{x}{\bar{x}}+\frac{\bar{x}}{x}-2\right) \\
& +\varphi(\bar{x}) \sum_{i=1}^{q} \pi_{i} b_{1, i}\left[k_{i}+2-\frac{\bar{x}}{x}-\frac{y_{1, i}}{\bar{y}_{1, i}} \frac{x}{\bar{x}} \frac{v}{\bar{v}}-\sum_{j=2}^{k_{i}} \frac{y_{j-1, i}}{\bar{y}_{j-1, i}} \frac{\bar{y}_{j, i}}{y_{j, i}}-\frac{y_{k_{i}, i}}{\bar{y}_{k_{i}, i}} \frac{\bar{v}}{v}\right] .
\end{aligned}
$$

We factorize $\frac{x-\bar{x}}{x \bar{x}}$ to get

$$
\begin{aligned}
\dot{V}_{E E} & =\frac{x-\bar{x}}{x \bar{x}}(a \bar{x} \varphi(x)-a x \varphi(\bar{x})+(a+u) \varphi(\bar{x})(x-\bar{x})) \\
& +\varphi(\bar{x}) \sum_{i=1}^{q} \pi_{i} b_{1, i}\left[k_{i}+2-\frac{\bar{x}}{x}-\frac{y_{1, i}}{\bar{y}_{1, i}} \frac{x}{\bar{x}} \frac{v}{\bar{v}}-\sum_{j=2}^{k_{i}} \frac{y_{j-1, i}}{\bar{y}_{j-1, i}} \frac{\bar{y}_{j, i}}{y_{j, i}}-\frac{y_{k_{i}, i}}{\bar{y}_{k_{i}, i}} \frac{\bar{v}}{v}\right] .
\end{aligned}
$$


Now there exists $\xi$ in the open interval $] x, \bar{x}[$ such that $\varphi(x)=\varphi(\bar{x})+(x-$ $\bar{x}) \varphi^{\prime}(\xi)$. Finally

$$
\begin{aligned}
\dot{V}_{E E} & =\frac{(x-\bar{x})^{2}}{x \bar{x}}\left(u \varphi(\bar{x})+a \bar{x} \varphi^{\prime}(\xi)\right) \\
& +\varphi(\bar{x}) \sum_{i=1}^{q} \pi_{i} b_{1, i}\left[k_{i}+2-\frac{\bar{x}}{x}-\frac{y_{1, i}}{\bar{y}_{1, i}} \frac{x}{\bar{x}} \frac{v}{\bar{v}}-\sum_{j=2}^{k_{i}} \frac{y_{j-1, i}}{\bar{y}_{j-1, i}} \frac{\bar{y}_{j, i}}{y_{j, i}}-\frac{y_{k_{i}, i}}{\bar{y}_{k_{i}, i}} \frac{\bar{v}}{v}\right] .
\end{aligned}
$$

Now the conclusion follows as in the proof of the one chain system.

Remark 3 When $u=0$ and $\varphi^{\prime}(\bar{x})<0$ the preceding computations show that if $\mathcal{R}_{0}>1$ the endemic equilibrium is locally asymptotically stable. When $u=1$ the condition $\beta \varphi(\bar{x})+\mu_{v} \varphi^{\prime}(\bar{x})<0$ is a sufficient condition for the asymptotic stability of the EE with $\mathcal{R}_{0}>1$.

\section{Applications}

In this section we will revisit the system considered in [15] modeling an HIV-1 infection.

$$
\left\{\begin{array}{l}
\dot{T}=s-d_{T} T-\left(1-n_{r t}\right) k V_{I} T \\
\dot{T}^{*}=k\left(1-n_{r t}\right) \int_{0}^{\infty} g_{n, b}(\tau) T(t-\tau) V_{I}(t-\tau) e^{-m \tau} d \tau-\delta T^{*} \\
\dot{V}_{I}=\left(1-n_{p}\right) N \delta T^{*}-c V_{I} \\
\dot{V}_{N I}=n_{p} N \delta T^{*}-c V_{N I} .
\end{array}\right.
$$

Where $s$ is the rate at which CD4 ${ }^{+}$T-cells are generated, $d_{T}$ their death rate, and $k$ is the constant rate of contact transmission. Once T-cells are infected they die at rate $\delta$ and produce $N$ new virus particles during their life. Virus particles are cleared at rate $c$. There is a continuous delay modeled by $g_{n, b}$ which is the Erlang distribution defined by (11). This model accounts for the effects of a drug therapy with reverse transcriptase inhibitor and a protease inhibitor. The term $n_{r t}$ is the effectiveness of the reverse transcriptase inhibitor in preventing new infections. The term $n_{p}$ is the efficacy of the protease inhibitor in percentage. The term $e^{-m \tau}$ accounts for cell that are infected at time $t$ but die before becoming productively infected $\tau$ time units later.

As in [15], the term $e^{-m \tau}$ can be absorbed into the delay kernel by defining

$$
\hat{b}=\frac{b}{1+m b} \quad \text { and } \quad \hat{k}=\frac{k}{(1+m b)^{n}}
$$

The mean of the gamma distribution $g_{n, \hat{b}}$ is $n \hat{b}$. Usually the mean for the 
intracellular delay is approximately known. Recent studies [3,38] give a figure between 1.8 and 2.6 days. If the mean delay is given by $\bar{\tau}$ we define $\hat{b}=\frac{\bar{\tau}}{n+m \bar{\tau}}$ and $\hat{k}$ is defined accordingly.

By the linear chain trick, the system (30) is equivalent to

$$
\left\{\begin{array}{l}
\dot{T}=s-d_{T} T-\left(1-n_{r t}\right) k V_{I} T, \\
\dot{y}_{1}=\hat{k}\left(1-n_{r t}\right) V_{I} T-\frac{1}{\hat{b}} y_{1}, \\
\dot{y}_{2}=\frac{1}{\hat{b}}\left(y_{1}-y_{2}\right), \\
\vdots \\
\dot{y}_{n}=\frac{1}{\hat{b}}\left(y_{n-1}-y_{n}\right), \\
\dot{T}^{*}=\frac{1}{\hat{b}} y_{n}-\delta T^{*} \\
\dot{V}_{I}=\left(1-n_{p}\right) N \delta T^{*}-c V_{I}, \\
\dot{V}_{N I}=n_{p} N \delta T^{*}-c V_{N I} .
\end{array}\right.
$$

The non-infectious virus $V_{N I}$ do not affect the dynamics of the other variable. Hence to study the stability of (31), the equation for the non-infectious virus $V_{N I}$ can be ignored.

Now the system (31) is a one chain system 12 with $n+1$ linear equations inserted between $T$ and $V_{I}$.

With our notations

$$
\begin{aligned}
& \beta=\left(1-n_{r t}\right) k, \\
& \gamma_{1}=\gamma_{2}=\cdots=\gamma_{n}=\frac{1}{\hat{b}}, \\
& \alpha_{1}=\ldots=\alpha_{n}=\frac{1}{\hat{b}}, \quad \gamma_{n+1}=\left(1-n_{p}\right) N \delta, \\
& \alpha_{n+1}=\delta, \\
& \mu_{v}=c, \\
& u=0 .
\end{aligned}
$$

Using the formula $(17)$ for $\mathcal{R}_{0}$ we obtain

$$
\mathcal{R}_{0}=\left(1-n_{r t}\right)\left(1-n_{p}\right) \frac{N s \hat{k}}{c d_{T}} .
$$

When $\hat{k}=k$, i.e., in the model for which the term $e^{-m \tau}$ is not introduced, the basic reproduction ratio does not depends on $n$. Applying Theorem 1 we deduce that either if $\mathcal{R}_{0} \leq 1$, then the viral free steady state is GAS, or else if 
$\mathcal{R}_{0}>1$, then the infected steady state is GAS. In [15] the conclusion was only local. In [15] the conclusion was related to the inequality $\eta_{c}<\eta_{\text {critical }}$ where $\eta_{c}=1-\left(1-n_{r t}\right)\left(1-n_{p}\right)$ and $\eta_{c r i t i c a l}=\frac{d_{T} c}{s N \hat{k}}$. This is clearly equivalent to the condition relative to $\mathcal{R}_{0}$.

When we account for cells dying before producing virions, with a mean delay $\bar{\tau}$, we get $\hat{k}=\frac{k}{\left(1+\frac{m \bar{\tau}}{n}\right)^{n}}$. In this case $\mathcal{R}_{0}$ is modified decreasingly. For $m \bar{\tau}$ fixed the increasing sequence $\left(1+\frac{m \bar{\tau}}{n}\right)^{n} \leq e^{m \bar{\tau}}$ converges monotonically towards $e^{m \bar{\tau}}$. In [15], $m$ is supposed small and even $m \ll 1$, hence multiplying $\mathcal{R}_{0}$ by $e^{-m \bar{\tau}}$ does not cause a great change, $\mathcal{R}_{0}$ is slightly lowered. If $\mathcal{R}_{0}$ is sufficiently far from 1 , then the infected equilibrium is not destabilized. To put it more precisely let us denote by $\mathcal{R}_{0}=\left(1-n_{r t}\right)\left(1-n_{p}\right) \frac{N s k}{c d_{T}}$ the basic reproduction ratio of the system without delay, if $\mathcal{R}_{0} e^{-m \bar{\tau}}>1$ then the endemic equilibrium is GAS for any continuous delay with kernel $g_{n, \hat{b}}$ where $\hat{b}=\frac{\frac{\bar{\tau}}{n}}{1+m \frac{\bar{\tau}}{n}}$. However for some values of $m>0$ and continuous delay, the endemic equilibrium can be theoretically destabilized. It is sufficient to take $\bar{\tau}$ sufficiently large, and to choose $n$ sufficiently large, in other words the variance of the gamma distribution $\frac{\bar{\tau}^{2}}{n}$ sufficiently small. The foregoing analysis makes no allowance for the biologically sensible values of the parameters. For example using the parameters of the references [2,38,3,13] we set $s=10$ (reference [13]), $d_{T}=$ 0.02 (reference [2]), $k=3.4310^{-5}$ (see [13]), $c=3$ (see [13,3]). With these values we obtain for $\mathcal{R}_{0}$ without treatment $\mathcal{R}_{0}=0.0057 \mathrm{~N}$. The number of infectious virus released $N$ is not known precisely, but values over $10^{3}$ are possible. If we choose $N=480$ (see [13]) we get $\mathcal{R}_{0}=2.74$ and if we choose $\left(1-n_{r t}\right)\left(1-n_{p}\right)=0.5$ we obtain, with treatment, $\mathcal{R}_{0}=1.37$. A destabilizing $\bar{\tau}$ should satisfy $m \bar{\tau}>0.316$. In [3] the death rate of latently infected $C D^{+} 4$ T-cell is assumed to be 0.02. If we conservatively choose this value for $m$, the destabilizing delay is $\bar{\tau}>15.8$ days. The viral generation time can be viewed as the time for an infected cell to produce $N$ new virions, this gives an estimation of the mean delay $\bar{\tau}$. Recent studies [38,3] give a figure between 1.8 days and 2.6 days which is incompatible with the destabilizing delay $\bar{\tau}=15.8$.

In [15] another model of standard form with delays is considered :

$$
\left\{\begin{array}{l}
\dot{T}=s-d_{T} T-\left(1-n_{r t}\right) k V T \\
\dot{T}^{*}=\bar{k}\left(1-n_{r t}\right) \int_{0}^{\infty} f_{1}(\tau) T(t-\tau) V(t-\tau) d \tau-\delta T^{*} \\
\dot{V}=\left(1-n_{p}\right) N \delta \int_{0}^{\infty} f_{2}(\tau) T^{*}(t-\tau) d \tau-c V .
\end{array}\right.
$$

In this model a second delay has been added. The introduction of this delay amounts to suppose that the virus particles $V$ appear $\tau$ time units after the 
infected cells began to produce. The linear chain trick can be applied provided $f_{1}$ and $f_{2}$ are reducible (convex combinations of Erlang distribution). For example if $f_{1}$ and $f_{2}$ are two Erlang distributions, then the system (32) is equivalent to

$$
\left\{\begin{array}{l}
\dot{T}=s-d_{T} T-\left(1-n_{r t}\right) k V T, \\
\dot{y}_{1}=\left(1-n_{r t}\right) k V T-\frac{1}{\hat{b}_{1}} y_{1}, \\
\dot{y}_{2}=\frac{\bar{k}}{k \hat{b}_{1}^{2}} y_{1}-\frac{1}{\hat{b}_{1}} y_{2}, \\
\dot{y}_{3}=\frac{1}{\hat{b}_{1}} y_{2}-\frac{1}{\hat{b}_{1}} y_{3}, \\
\vdots \\
\dot{y}_{n}=\frac{1}{\hat{b}_{1}} y_{n-1}-\frac{1}{\hat{b}_{1}} y_{n}, \\
\dot{T}^{*}=\frac{1}{\hat{b}_{1}} y_{n}-\frac{1}{b_{2}} T^{*}, \\
\dot{T}_{1}^{*}=\frac{1}{b_{2}} T^{*}-\frac{1}{b_{2}} T_{1}^{*}, \\
\vdots \\
\dot{T}_{n^{\prime}}^{*}=\frac{1}{b_{2}} T_{n^{\prime}-1}^{*}-\frac{1}{b_{2}} T_{n^{\prime}}^{*}, \\
\dot{V}=\left(1-n_{p}\right) N \frac{1}{b_{2}} T_{n^{\prime}}^{*}-c V .
\end{array}\right.
$$

With $b_{2}=n^{\prime} / \bar{\tau}_{2}$, if the mean delay is $\bar{\tau}_{2}$ for the second delay. This is again a one chain system. We suppose as in [15] that $\bar{k}$ is independent of $n$. Using the formula for $\mathcal{R}_{0}$ we obtain again

$$
\mathcal{R}_{0}=\left(1-n_{r t}\right)\left(1-n_{p}\right) \frac{N s \bar{k}}{c d_{T}}
$$

We obtain a result similar to the one of [15], but with two continuous delays: since $\mathcal{R}_{0}$ does not depend on $n$ and $n^{\prime}$, the global stability of the equilibria is not modified by the delays. If $\bar{k}$ depends on $n$, then an analysis similar to the one done for the preceding example can be done.

\section{Conclusions}

We have proved stability for general within-host parasitic systems. These systems can be interpreted as systems with continuous delays that are modeled by gamma distributions or convex combinations of gamma distributions. We have computed the basic reproduction ratio $\mathcal{R}_{0}$ for these models. Conditions for global asymptotic stability of the equilibria have been given. In some cases these conditions are NSC. The stability is strongly related to $\mathcal{R}_{0}$. We have applied these results to some classical models of HIV-1 infection. Our results should be applicable to general set of delay differential equations. 


\section{References}

[1] A. Perelson, Modeling the interaction of the immune system with HIV, in: C. Castillo-Chavez (Ed.), Mathematical and statistical approaches to AIDS epidemiology with HIV, Springer-Verlag, 1989, pp. 350-370.

[2] A. S. Perelson, D. E. Kirschner, R. De Boer, Dynamics of HIV infection of CD4+ T cells., Math Biosci 114 (1993) 81-125.

[3] A. S. Perelson, P. W. Nelson, Mathematical analysis of HIV-1 dynamics in vivo, SIAM Rev. 41 (1) (1999) 3-44 (electronic).

[4] R. M. Anderson, R. M. May, S. Gupta, Non-linear phenomena in host-parasite interactions., Parasitology 99 Suppl (1989) S59-79.

[5] R. M. Anderson, Complex dynamic behaviours in the interaction between parasite population and the host's immune system., Int J Parasitol 28 (1998) $551-66$.

[6] C. Hetzel, R. M. Anderson, The within-host cellular dynamics of bloodstage malaria: theoretical and experimental studies., Parasitology 113 ( Pt 1) (1996) $25-38$.

[7] M. A. Nowak, S. Bonhoeffer, A. Hill, R. Boehme, H. Thomas, H. McDade, Viral dynamics in hepatitis B virus infection., Proc. Natl Acad. Sci. U. S. A. 93 (1996) 4398-4402.

[8] M. A. Nowak, C. Bangham, Population dynamics of immune responses to persistent viruses., Science 272 (1996) 74-79.

[9] R. J. Payne, M. A. Nowak, B. S. Blumberg, The dynamics of hepatitis B virus infection, Proc Natl Acad Sci U S A 93.

[10] M. A. Nowak, R. M. May, virus dynamics. Mathematical Principles of Immunology and Virology, Oxford University Press, 2000.

[11] L. Molineaux, K. Dietz, Review of intra-host models of malaria., Parassitologia 41 (1-3) (2000) 221-31.

[12] J. A. Jacquez, C. P. Simon, Qualitative theory of compartmental systems with lags, Math. Biosci. 180 (2002) 329-362.

[13] P. W. Nelson, J. Murray, A. S. Perelson, A model of HIV-1 pathogenesis that includes an intracellular delay., Math. Biosci. 163 (2000) 201-215.

[14] J. E. Mittler, B. Sulzer, A. U. Neumann, A. S. Perelson, Influence of delayed viral production on viral dynamics in HIV-1 infected patients., Math. Biosci. 152 (2) (1998) 143-163.

[15] P. W. Nelson, A. S. Perelson, Mathematical analysis of delay differential equation models of HIV-1 infection., Math. Biosci. 179 (1) (2002) 73-94. 
[16] J. A. Jacquez, Modeling with Compartments, BioMedware, 1999.

[17] J. A. Jacquez, Compartmental Analysis in Biology and Medicine, BioMedware, 1996.

[18] N. MacDonald, Time lags in Biological Models, Lecture Notes in Biomathematics No 27, Springer-Verlag, Berlin, 1978.

[19] D. Cox, H. Miller, The Theory of Stochastic Processes, Chapman and Hall, 1965.

[20] Z. Grossman, M. Feinberg, K. Kuznetsov, D. Dimitrov, W. Paul, HIV infection : how effective is drug combination treatment, Immunol. Today 19 (1998) 528532 .

[21] A. L. Lloyd, Destabilization of epidemic models with the inclusion of realistic distributions of infectious periods., Proc R Soc Lond B Biol Sci 268 (1470) (2001) 985-93.

[22] A. L. Lloyd, The dependance of viral parameter estimates on the assumed viral life cycle : limitations of studies of viral load data, Proc R Soc Lond B Biol Sci 268 (2001) 847-854.

[23] A. L. Lloyd, Realistic distributions of infectious periods in epidemic models: changing patterns of persistence and dynamics., Theor Popul Biol 60 (2001) $59-71$.

[24] P. van den Driessche, J. Watmough, Reproduction numbers and sub-threshold endemic equilibria for compartmental models of disease transmission, Math. Biosci. 180 (2002) 29-48.

[25] O. Diekmann, J. A. P. Heesterbeek, J. A. J. Metz, On the definition and the computation of the basic reproduction ratio $R_{0}$ in models for infectious diseases in heterogeneous populations, J. Math. Biol. 28 (4) (1990) 365-382.

[26] E. D. Sontag, Mathematical Control Theory, Deterministic Finite Dimensional Systems, no. 6 in Texts in Applied Mathematics, Springer-Verlag, 1990.

[27] D. G. Luenberger, Introduction to Dynamic Systems. Theory, Models, and Applications., John Wiley \& Sons Ltd., 1979.

[28] E. Lee, L. Markus, Foundations of Optimal Control Theory, John Wiley \& Sons Ltd., 1967.

[29] J. A. Jacquez, C. P. Simon, Qualitative theory of compartmental systems, SIAM Rev. 35 (1) (1993) 43-79.

[30] E. Beretta, Y. Takeuchi, Global stability of lotka-volterra diffusion models with continuous time delay., SIAM J. Appl. Math. 48 (1988) 627-651.

[31] H. W. Hethcote, The mathematics of infectious diseases, SIAM Rev. 42 (4) (2000) 599-653 (electronic). 
[32] O. Diekmann, J. A. P. Heesterbeek, Mathematical Epidemiology of Infectious Diseases, model building, analysis and interpretation, Wiley Series in Mathematical and Computational Biology, John Wiley \& Sons Ltd., Chichester, 2000 .

[33] G. J. Butler, P. Waltman, Persistence in dynamical systems, J. Differ. Equations 63 (1986) 255-263

[34] J. K. Hale, Asymptotic behavior of dissipative systems, A.M.S. Providence, 1988.

[35] M. Nagumo, Über die lage der integralkurven gewohnlicher differentialgleichungen, Proc. Phys.-Math. Soc. Jap. 24, (1942) 551-559.

[36] N. P. Bhatia, G. P. Szegö, Dynamical systems: Stability theory and applications, Springer-Verlag, 1967.

[37] J. LaSalle, Stability theory for ordinary differential equations., J. Differ. Equations, 41 (1968) 57-65.

[38] A. S. Perelson, P. W. Nelson, Modeling viral infections, in: An introduction to mathematical modeling in physiology, cell biology, and immunology (New Orleans, LA, 2001), Vol. 59 of Proc. Sympos. Appl. Math., Amer. Math. Soc., Providence, RI, 2002, pp. 139-172.

[39] L. Schwartz, Methods of Mathematical Physics, Addison-Wesley, Reading Mass., 1964.

[40] P. De Leenheer, H. L. Smith, Virus dynamics: A global analysis., SIAM J. Appl. Math. 63 (4) (2003) 1313-1327. 